\title{
Development of low-density coatings for soft X-ray reflectivity enhancement for ATHENA and other missions
}

Vincenzo Cotroneo, Ricardo Bruni, Thorsten Döhring, Desiree Ferreira, Eugenio Gibertini, et al.

Vincenzo Cotroneo, Ricardo Bruni, Thorsten Döhring, Desiree Ferreira, Eugenio Gibertini, Peter Henriksen, Luca Magagnin, Sonny Massahi, Giovanni Pareschi, Suzanne Romaine, Leandra Sethares, Giorgia Sironi, Daniele Spiga, Gianpiero Tagliaferri, Giuseppe Valsecchi, "Development of low-density coatings for soft x-ray reflectivity enhancement for ATHENA and other missions," Proc. SPIE 11852, International Conference on Space Optics - ICSO 2020, 118524P (11 June 2021); doi: 10.1117/12.2599803

SPIE Event: International Conference on Space Optics - ICSO 2021, 2021, Online Only 


\section{International Conference on Space Optics-ICSO 2020}

Virtual Conference

30 March-2 April 2021

Edited by Bruno Cugny, Zoran Sodnik, and Nikos Karafolas
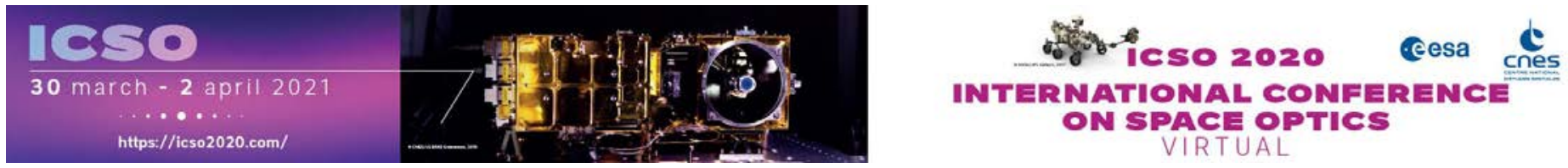

\section{Development of low-density coatings for soft $x$-ray reflectivity enhancement for ATHENA and other missions}

\section{Cesa isoporecestings denes}




\title{
Development of low-density coatings for soft X-ray reflectivity enhancement for ATHENA and other missions
}

\author{
Vincenzo Cotroneo ${ }^{1}$, Ricardo Bruni ${ }^{2}$, Thorsten Döhring ${ }^{3}$, Desiree Ferreira ${ }^{4}$, Eugenio Gibertini ${ }^{5}$, \\ Peter Henriksen ${ }^{4}$, Luca Magagnin ${ }^{5}$, Sonny Massahi ${ }^{4}$, Giovanni Pareschi ${ }^{1}$, Suzanne Romaine ${ }^{2}$, \\ Leandra Sethares ${ }^{2}$, Giorgia Sironi ${ }^{1}$, Daniele Spiga ${ }^{1}$, Gianpiero Tagliaferri ${ }^{1}$, Giuseppe Valsecchi ${ }^{6}$ \\ ${ }^{1}$ INAF-Osservatorio Astronomico di Brera, Via E. Bianchi 46, 23807 Merate, Italy \\ ${ }^{2}$ Center for Astrophysics | Harvard and Smithsonian, 60 Garden St, Cambridge, MA, 02138, USA \\ ${ }^{3}$ Technische Hochschule Aschaffenburg, Würzburger Str. 45, D-63743 Aschaffenburg, Germany \\ ${ }^{4}$ DTU-space, Juliane Maries Vej 30, DK-2100 Copenhagen (Denmark) \\ ${ }^{5}$ Politecnico di Milano, Dipartimento di Chimica, Materiali e Ingegneria Chimica "Giulio Natta", via \\ Mancinelli 7, I-20131 Milano, Italy \\ ${ }^{6}$ Media Lario S.r.l., Località Pascolo, I-23842 Bosisio Parini (LC), Italy
}

\begin{abstract}
Metallic coatings made of precious metals (e.g. Ir, Pt or Au) with high atomic number $\mathrm{Z}$ have been traditionally employed at grazing incidence for X-rays reflection and focalization. These materials offer a range of reflection extended to higher energies, but also present a series of absorption edges, which limit the reflectivity in the $2-4 \mathrm{keV}$ band and below.

Therefore the search for alternative coatings, able to improve the reflection in the soft energy range, is particularly relevant for the development of future telescopes, like ATHENA (ESA), Lynx (NASA) and eXTP (CAS).

Low-Z overcoatings (e.g. carbon or $\mathrm{B}_{4} \mathrm{C}$ ), applied on top of the high- $\mathrm{Z}$ metallic layer, can enhance the reflectivity in the softer band (mainly below $2 \mathrm{keV}$ ), but conventional deposition methods for these materials are not easily compatible with some of the mainstream technologies for mirror fabrication (notably, the silicon pore optics that will be used for the ATHENA X-ray mission which is being implemented by ESA).

In this work we discuss novel solutions (carbon-like overcoatings realized by dip coating or vapor phase deposition), which can be particularly convenient for the application to ATHENA and to future telescopes.
\end{abstract}

Keywords: X-ray Optics, light coatings, soft X-rays reflectivity, ATHENA, grazing incidence optics

\section{INTRODUCTION}

Wolter I type X-ray telescopes focus light by total reflection at grazing incidence mirrors, in general implemented by means of a set of concentric confocal nested surfaces. The most suitable materials for the reflection of X-rays over a broad energy band are high-Z materials (tipically $\mathrm{Au}, \mathrm{Ir}, \mathrm{Pt}$ ), that are applied as reflecting coating on the optics substrate.

Several technologies are available and used for the fabrication of X-ray telescopes. Most widespread technologies are nickel electroformed replica monolithic optics with gold reflecting coating (used also as a separation agent in the replication process), and platinum or iridium coatings, deposited on figured glass substrates ${ }^{1,2}$ (monolithic or segmented). Iridium coating on silicon plates is also the technology that will be used for the ATHENA (ESA) and Lynx (NASA) missions ${ }^{3-5}$.

The high-density metals used in this kind of applications, while quite effective up to a higher energy, because of the inverse relation between critical angle for total reflection and density, present however low-energy absorption edges, which cause abrupt reduction of reflectivity around their characteristic energies. It has been proposed the use of carbon (also in Diamond-like form) or $\mathrm{B}_{4} \mathrm{C}$ as overcoatings on top of the metal layers in order to increase the reflectivity in correspondence of the absorption edges of the metal layer ${ }^{6-11}$.

In this paper, we discuss the use of novel types of overcoatings, based on the concept of "dip" deposition, i.e. that can be realized by immersing a substrate (or, when possible, a finite optics) in a precursor solution, and letting the coating material 
deposit on the surface. We have considered substrates made of nickel with gold coatings, representative of nickel-replica optics (used in past missions like XMM, Swift and eRosita, and futures projects like eXTP ${ }^{12}$ ), and silicon plates, coated with iridium and with a thin $(2-10 \mathrm{~nm})$ layer of chromium on top of it. These last substrates are representative of silicon pore optics, like the ones foreseen for the ATHENA mission. Even if the baseline configuration for ATHENA is based on simple iridium coatings, we have also considered a thin chromium overcoating (deposited by sputtering), as this can considerably enhance the reflectivity of the mirror at the cost of a relatively small change in the optics manufacturing process $^{13,14}$. It has to be noticed that chromium is also used as a binding layer, as such is already present in the sputtering chamber used for the reflecting coating deposition for ATHENA. As such, the manufacturing challenge is a mere issue of control of thickness and film structure, in particular the micro-roughness, and film internal stress. It has to be noticed that chromium can be a difficult material to deposit in thick layers (films tend to have high stress and roughness), however, as far as we know, stress and roughness of very thin (few nanometers) are not commonly studied, and we may reasonably expect these problems to be smaller than for thicker films. We have deposited films of this kind in two different facilities with different sputtering methods. We first discuss the theoretical performances expected from this kind of coatings and overcoatings, then we describe the deposition and the characterization of the deposited coatings.

\section{DIP DEPOSITION OF OVERCOATINGS}

"Dip" coatings considered in this work, belong to two different categories: self-regulating coatings (notably selfassembling monolayers, SAMs), which grow ordered structures with definite thickness or, in alternative, indefinite-growth coatings, for which the sedimentation of structures happens continuously and the final structure of the film is regulated by the characteristics of the precursor solution and by deposition conditions and time. While not investigated in this work, these coatings can also be deposited by other methods as e.g. physical (PVD) and chemical vapor deposition (CVD).

The overcoating material chemically binds to the top metal layer of the substrate, which determines the choice of the material and precursor solution for the film to be deposited.

We have considered two types of reflecting layers and corresponding overcoatings:

- $\mathrm{Ir} / \mathrm{Cr}$ coatings, which are an extension of the Ir coatings planned for ATHENA. In this case the baseline coating for the mission is Ir, but it was recently proposed that a thin layer of chromium can enhance the reflectivity over the band $2-4 \mathrm{keV}$

- Au coatings, that have been applied to many missions based on nickel replica optics (e.g. XMM, eRosita on board of Spectrum-Roentgen-Gamma), and are planned on future missions with moderate angular resolution, like eXTP.

To obtain a surface quality representative of the two missions, we used silicon plates provided by the company Cosine (www.cosine.nl) on top of which we applied iridium and chromium coatings; and fragments of gold-coated nickelreplicated shells provided by the company Media Lario, or flat samples realized with the same process.

The coatings selected for the liquid-phase deposition on top of the metallic layers were polydopamine for the $\mathrm{Ir} / \mathrm{Cr}$ coatings and a self-assembled bilayer of thiol and silane compounds, for the gold coatings. Both of these materials have a known compatibility with the underlying material, showing features of good adhesion and smooth surface. They are commonly used in a broad range of research and industrial applications; biotechnologies, electronics, MEMS, among the others. In particular, thiol coatings on Au are widely employed as Au capping agent in Au nanoparticles synthesis, electronics and sensors. $^{15-17}$
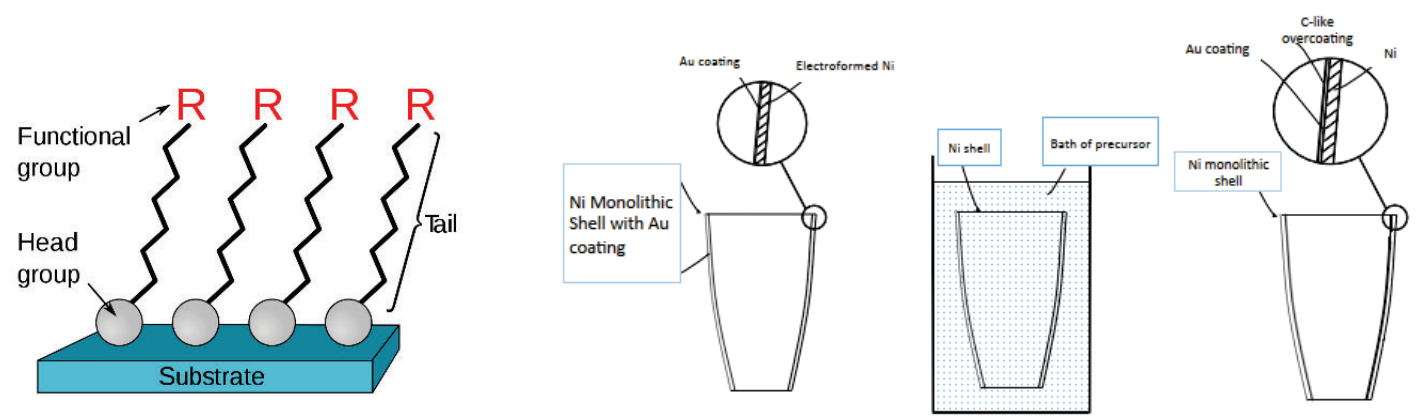

Figure 1: Schematics of adhesion mechanisms for SAM coatings ${ }^{18}$ (left) and process of deposition on a nickel monolithic shell with $\mathrm{Au}$ coating produced by replication (right). 
The mechanism for which these substances form ordered structures on the surface of a substrate, is shown in fig. 1 for SAMs, which are composed of long chains with different properties at the head and the tail. In the case depicted in figure 1 , the head is chemisorbed on the gold surface while molecules orient along a direction transversal to the surface, forming an ordered layer, whose thickness is determined by the length of the chain. Compounds of this kind, when deposited in ordered structures, are self-limiting, being their growth inhibited once the free sites on the underlying layer are occupied. If the thickness is lower than desired, a compound can be chosen where the tail is functionalized, and a second substance, with affinity for the free ends, is used to build a second layer. Dopamine follows a similar mechanism, with molecules adhering to substrates, then polymerizing and forming intra-molecular bonds, giving stability to the coating, even for looser bounds to the surface. Indeed for thiol the adhesion to chromium is difficult because of the presence of a stable oxidized layer, making polydopamine a more appropriate choice.

\section{EXPECTED PERFORMANCES}

Intuitively, we can say that lower energies are reflected by the top low-Z overcoating, while the higher energies penetrate deeper and are reflected by the metallic coating, thus preventing the absorption of low-energy radiation in correspondence of K-edges of the underlying metallic coating ${ }^{19}$. This picture is however a simplification; as interference and change of phase at the interfaces must be kept into account and the coatings must be simulated using a correct physical model. We have simulated the coating reflectivity by means of IMD software ${ }^{20}$ on the base of the coating chemical composition and expected density $\left(1.0 \mathrm{~g} / \mathrm{cm}^{3}\right.$ for all carbon based coatings, as nominal values from datasheet). Chromium has been modeled as chromium-oxide, as a more realistic form for such a thin layer.
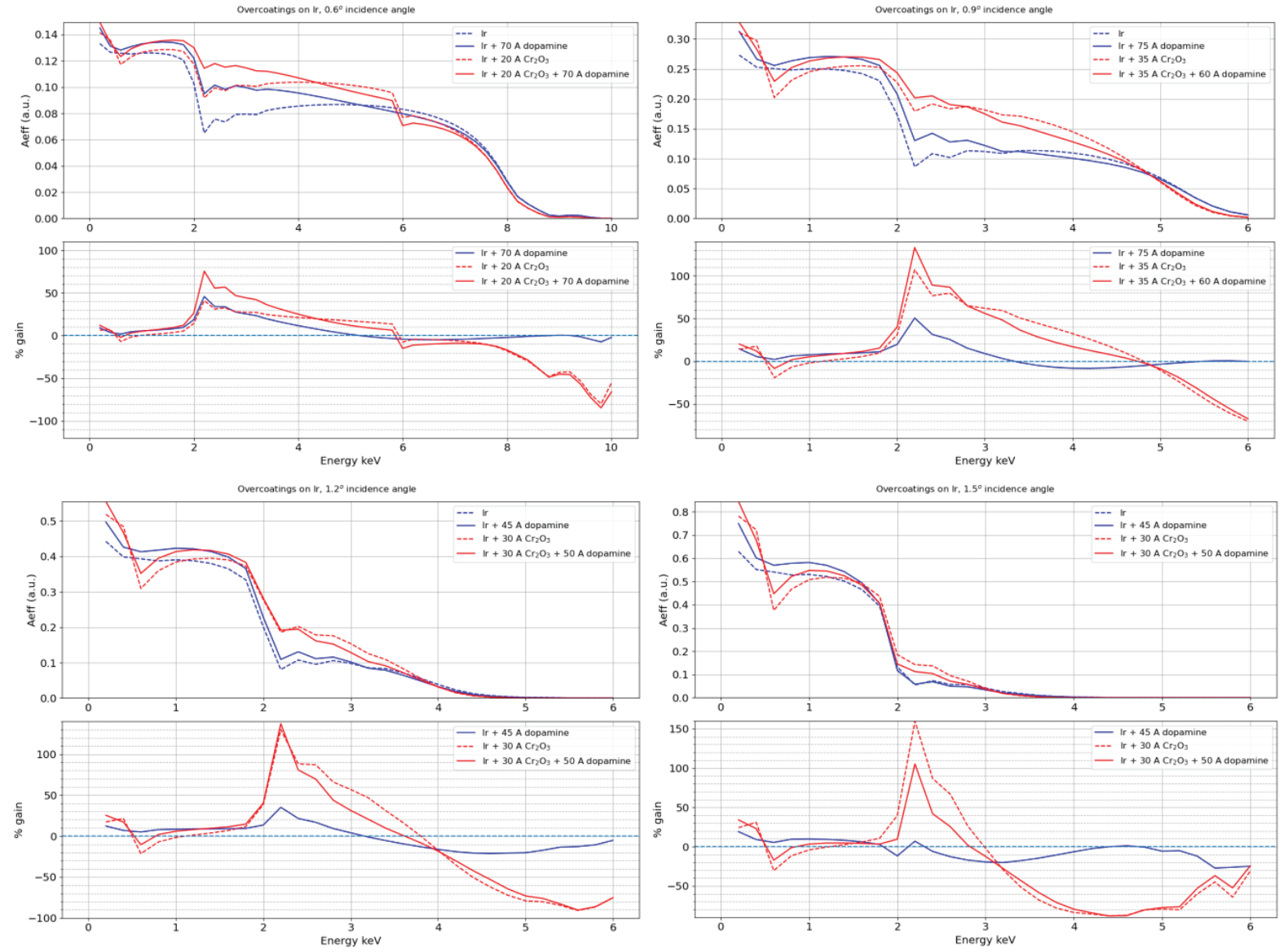

Figure 2: Each subfigure shows the effective area for a shells at a given angle with different combinations of iridium and chromium coatings and dopamine overcoatings, as fraction of the collecting area of a shell at largest angle of $1.5^{\circ}$ : blue lines correspond to iridium only coatings, while iridium+chromium coatings are represented by red lines. Continuous lines have a dopamine layer on top, while dashed lines have no overcoating. Bottom panels of each subplot show the percentual gain over the baseline coating (iridium monolayer). 

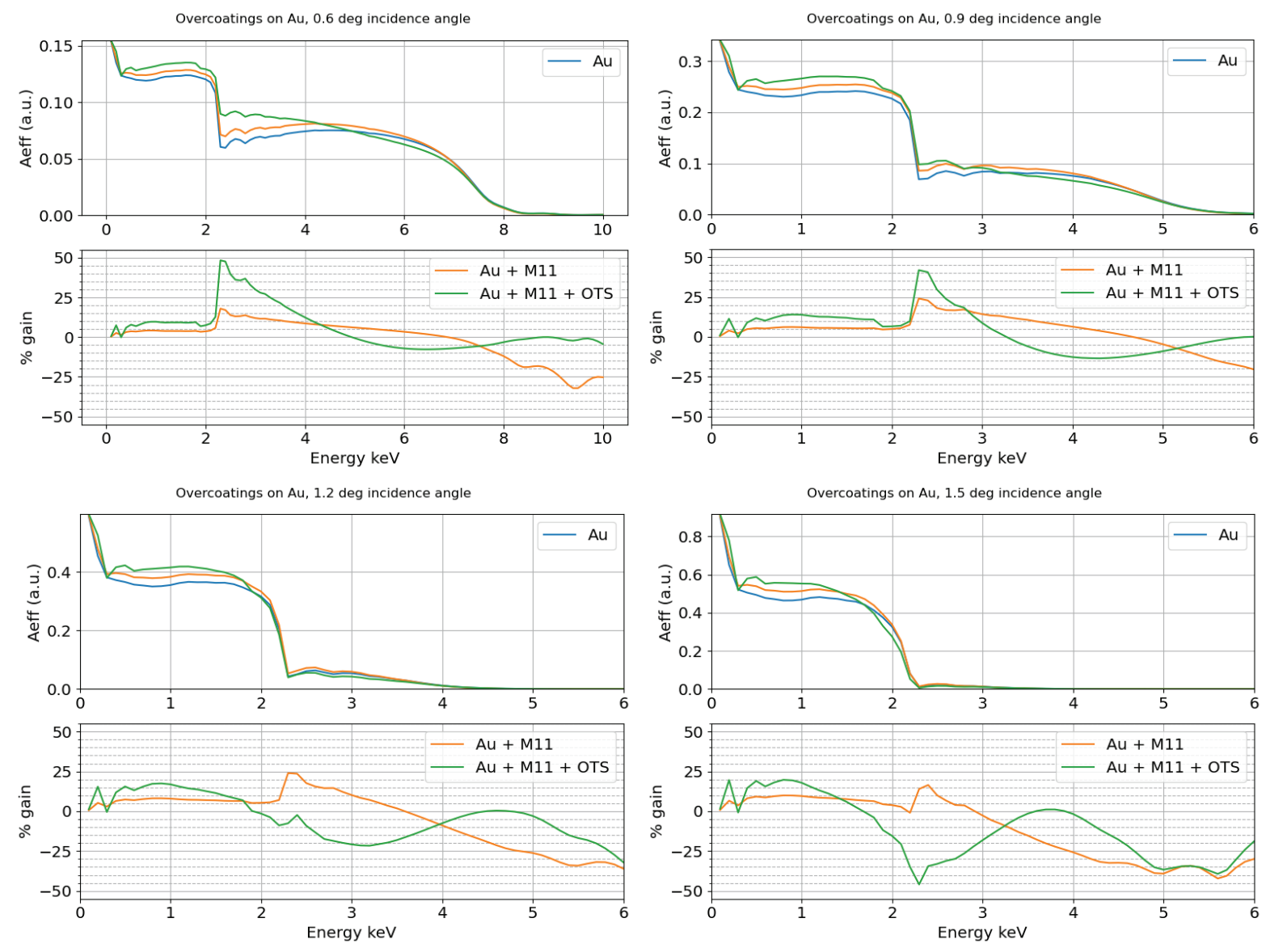

Figure 3: Expected gain for SAM overcoatings on top of a gold reflecting surface at four representative angles. Combinations with the first layer only ( $2.5 \mathrm{~nm}$ of M11) and with both layers $(2.5 \mathrm{~nm} \mathrm{M11}+4.5 \mathrm{~nm}$ OTS) are considered. Effective Area are expressed as fraction of collecting area of a shell with incidence angle of $1.5^{\circ}$.

The effective area of different combinations of coatings is shown in fig. 2 and 3 for a few representative angles in the typical range of interest for X-ray telescopes (grazing incidence angle $0.3^{\circ}-1.5^{\circ}$, energy $0-10 \mathrm{keV}$ ), respectively for iridium/chromium and gold coated substrates. Effective areas are shown in arbitrary units, where the unit is represented by the collecting area of a shell at the largest angle $\left(1.5^{\circ}\right)$, in this way the areas maintain the correct proportion between the different shells having same angles in a telescope of fixed shell length. Indeed, for an X-ray telescope in Wolter I geometry, formed by a large number of concentric shells, the reflection angle $\alpha_{\mathrm{i}}$ on each of them is given by $\alpha_{i}=\frac{1}{4}$ atan $\frac{R_{i}}{F}$ with $\mathrm{R}_{\mathrm{i}}$ radius of shell $i$ at intersection plane and $\mathrm{F}$ focal length. As such, each change in reflectivity is amplified by the square, while the collecting area is, for a given shell height, approximately proportional to the square of the radius (being the projected area of a shell a circular corona of width $\Delta R=H \tan \alpha$, with $\mathrm{H}$ shell length and angle $\alpha$ proportional to radius in small angle approximation).

Plots show that the choice of coatings, and of coating parameters, is a trade-off. For most of the mirror shells of a given X-ray telescope, increased reflectivity in a given energy interval, can correspond to a decrease in other parts of the spectrum, with the relevance of each gain, positive or negative, and the overall impact on telescope area, depending on the details of the telescope geometry. However it is also apparent how in many cases, the proposed combinations of coatings can give an increase of effective area between $10 \%$ and $50 \%$ in spectral regions of scientific relevance, with little or no loss (i.e. large fractional reductions of effective area happen in regions where the shell effective area is negligible).

In particular, for iridium-based mirrors, the use of chromium can give a large enhancement of effective area in the region just above the iridium $\mathrm{M}$ absorption edge (2.04-3.17 keV), but depletes the low energy reflectivity at chromium L-edge 
(0.5-0.69 keV) and, for very small angles, above the K-edge at $5.99 \mathrm{keV}$. Thicker layers of chromium enhance the gain just above the iridium $\mathrm{M}$ edge, but lead to a loss in the higher part of this range. Carbon-based coatings can compensate, partially or completely, the depletion below $2 \mathrm{keV}$ and, for the smaller angles, even above this energy, making it possible to use a thinner layer of chromium and to reduce the loss above the chromium edge at $6 \mathrm{keV}$ (see e.g. first panel of figure 2).

\section{COATINGS AND OVERCOATINGS ON SILICON SUBSTRATES}

\section{Deposition and characterization of metallic layers}

Silicon plates provided by the company Cosine, representative of the substrates that will be used for the fabrication of ATHENA's optics, have been coated at two facilities; the DC magnetron sputtering chamber at Smithsonian Astrophysical Observatory (SAO) and the DC magnetron sputtering chamber at Aschaffenburg University (THAB). The two facilities differ for the different control of minimal thickness and for the different optimization of the process for these coatings, which are routinely manufactured in both facilities ${ }^{21-29}$, which also have developed methods to control stress in this kind of films ${ }^{24-25}$. The SAO facility has a finer control of the deposited thickness and can produce nanometric or subnanometric thickness films made of sputtered metals layers. For the facility at THAB, slightly thicker layers of chromium had to be used in order to ensure a uniform coverage of the sample.

Iridium was deposited directly on silicon at SAO with a thickness of $30 \mathrm{~nm}$. The thin chromium overcoating with target thickness of $2 \mathrm{~nm}$ was deposited on some of the samples (others were kept with only iridium for surface characterization). Samples were characterized in X-rays at $8.04 \mathrm{keV}$ at SAO, giving thickness value into $10 \%$ deviation from target value for iridium and 20\% for chromium. Coatings deposited at THAB use a $40 \mathrm{~nm}$ binding layer below the iridium to ensure adhesion to the substrate, as well as layer stress compensation ${ }^{22}$, while the thin chromium overcoating was deposited with thickness values of 6 and $10 \mathrm{~nm}$ for different batches of samples, over a $30 \mathrm{~nm}$ thick layer of iridium.
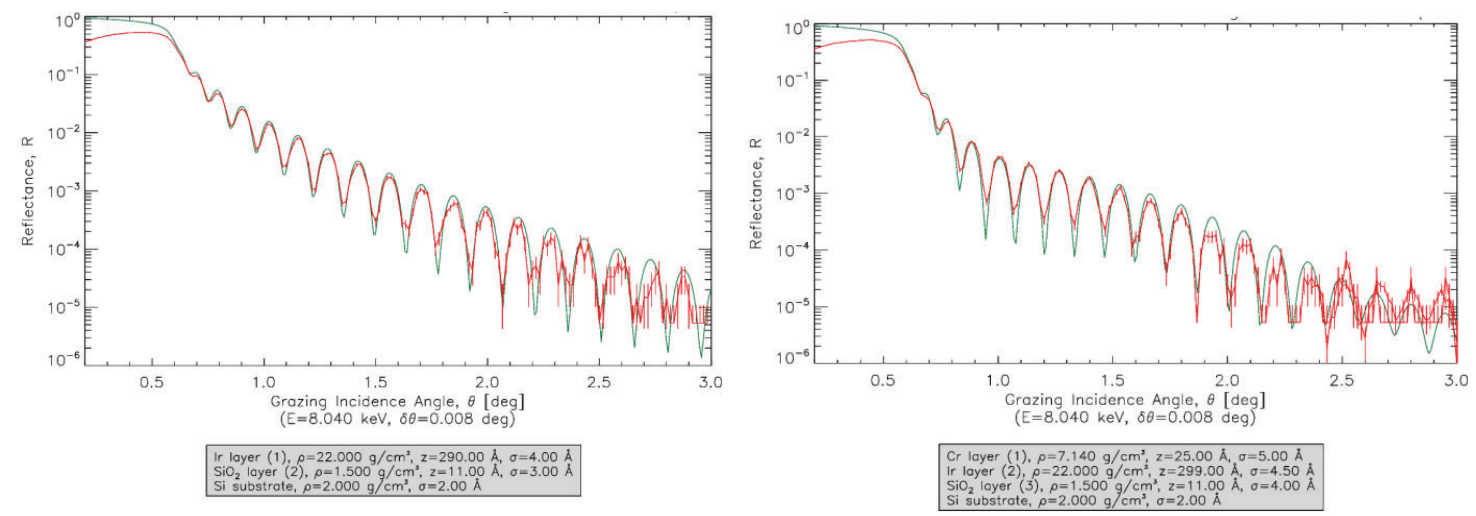

Figure 4: Example of data and procedure used to characterize samples at SAO. Thickness, roughness and density can be fit in unambiguous way from X-ray reflectivity data on angular scans at $8.04 \mathrm{keV}$.

Substrates from both deposition facilities with both iridium only and the combination iridium + chromium were characterized using surface metrology tools at OAB: atomic force microscope (AFM) on three scales $(1,10$ and $50 \mu \mathrm{m})$, and MicroFinish Topographer phase measuring interferometer (MFT) with 10x objective (image size $0.9 \mathrm{~mm}$ for 1024 pixels). The power spectral density (PSD) was extracted from topography data for all samples and joined to give an overall power spectrum.

PSD data on different scales have shown a good alignment and correspondence over common frequency intervals, with similar surface quality for all coatings. There is a slight increase of surface roughness along the stack of layers from bottom to top (fig. 5, table 1), with consistent values obtained from X-ray data on SAO samples. Notably all coatings are in the range accepted for X-ray reflective surfaces, being the surface roughness on combined AFM scales lower than $0.5 \mathrm{~nm}$ and comparable with the values for the bare substrates. A slightly higher value was found for the thinner chromium coatings realized at THAB (6 $\mathrm{nm}$, near the lower limit for the deposition process), which might indicate an incomplete growth of the layer. A detailed views of rms calculated values over different frequency intervals for the different combinations of coatings and overcoatings is shown in table 1. 


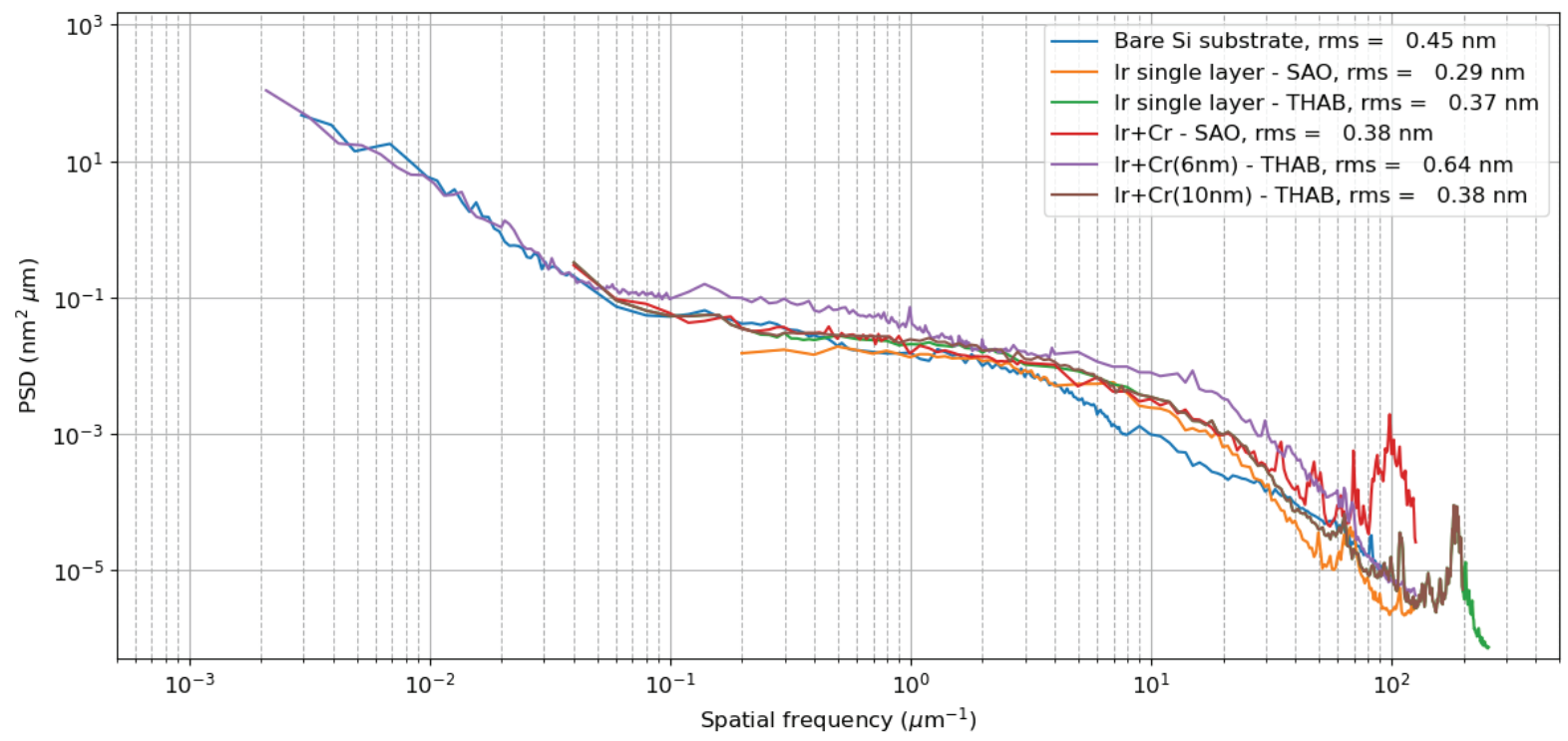

Figure 5: Comparison of substrates and evolution of PSD for the deposition of each layer. Values of rms roughness in legend are calculated as square root of PSD integrated over the largest frequency range available from data. Peaks at high end of the spectrum are due to environmental and electronic noise (they repeat with same shape over different measurement ranges according to the scan size and sampling rate) and can be shown to have a negligible impact on the rms calculation.

Table 1: values of rms roughness estimated over extended frequency range of spatial frequencies. Values are derived from surface metrology data on different samples and areas, by integrating of the PSD. The largest frequency range available for each coating type is listed, together with a common frequency range, roughly corresponding to the full AFM scale. The range of values obtained over different samples from fit of X-ray reflectivity data at $8.04 \mathrm{keV}$ (for only metallic coatings at $\mathrm{SAO}$, measurements at this energy are not sensitive to overcoatings).

\begin{tabular}{lccc}
\hline Coating & \multicolumn{2}{c}{ rms from PSD $(\mathrm{nm})$ over freq range } & $\begin{array}{c}\text { rms from X-ray } \\
\text { reflectivity fit } \\
\text { at } 8.04 \mathrm{keV} .\end{array}$ \\
\hline $\mathrm{Si}$ Bare & full range [freq. & {$[0.10: 100]$} & $0.25-0.35$ \\
$\mathrm{Si}+\mathrm{Ir} \mathrm{SAO}$ & $0.46[0.003: 100]$ & 0.22 & $0.35-0.45$ \\
$\mathrm{Si}+\mathrm{Ir}$ THAB & $0.30[0.1: 128]$ & 0.30 & \\
$\mathrm{Si}+\mathrm{Ir}+\mathrm{Cr} \mathrm{SAO}$ & $0.38[0.020: 255]$ & 0.36 & $0.4-0.5$ \\
$\mathrm{Si}+\mathrm{Ir}+\mathrm{Cr}$ THAB $6 \mathrm{~nm}$ & $0.39[0.02-128]$ & 0.36 & \\
$\mathrm{Si}+\mathrm{Ir}+\mathrm{Cr}$ THAB $10 \mathrm{~nm}$ & $0.75[0.001: 128]$ & 0.52 & \\
\hline Dopamine $4 \mathrm{~h}$ & $0.39[0.02-200]$ & 0.37 & \\
Dopamine 2.5 d & $0.88[0.100-100]$ & 0.87 & \\
Dopamine 24 h & $6.46[0.06-255]$ & 5.8 & \\
Dopamine 24 h (range) & $0.76[0.003: 100]$ & 0.69 & \\
\hline
\end{tabular}




\section{Dopamine overcoating}

We have deposited polydopamine overcoatings on THAB coated substrates using different immersion times. Polydopamine coatings are obtained by dopamine $\left(\mathrm{C}_{8} \mathrm{H}_{11} \mathrm{NO}\right)$ auto-polymerization ${ }^{30-31}$ or oxidative polymerization ${ }^{32}$ in alkaline solution. Here, polydopamine coatings were obtained according to procedure adapted from literature ${ }^{32}$. A $20 \mathrm{mM}$ dopamine hydrochloride solution and $20 \mathrm{mM}$ ammonium persulfate (APS) in $10 \mathrm{mM}$ TRIS-buffer (pH 8.5) was prepared and samples were immersed in solution at ambient temperature. Density is usually around $1 \mathrm{~g} / \mathrm{cm}^{3}$.

Times between 4 hours and 2.5 days were used to develop the deposition process. Film growth was apparent from the change of discoloration of the sample and surface structure. Measurement of dopamine thickness by means of ellipsometry is challenging because of the difficult determination of the optical constants for the material. We scratched the coating by using a copper tip and used the MFT profilometer to measure the step and determine the coating thickness. A deposition time of $24 \mathrm{~h}$ was eventually considered optimal to obtain the appropriate level of thickness and surface roughness and used for our "best-effort" samples. The method used for thickness determination has high uncertainty, but measurements consistently indicate a thickness between 5 and $10 \mathrm{~nm}$.

We have measured surface topography using same methods described for the $\mathrm{Cr}$ and Ir surfaces. Results show an increase of surface roughness from the 4 hours to the 2.5 days immersion time. The final sample deposited for $24 \mathrm{~h}$ has a rms roughness that is better or equal to the one measured on any of the two reference samples, with PSD comparable to the base substrate. AFM images are shown in fig. 6 and 7, including a large area scan (100 $\mu \mathrm{m}$ size), which gives an idea of the uniformity of the coating. There is some variability in data taken in different positions or on different samples. We provide an estimate of this variability by indicating a realistic interval of PSDs, obtained from all data, and a PSD that we consider a representative estimate of the coating quality (fig. 8, 9).

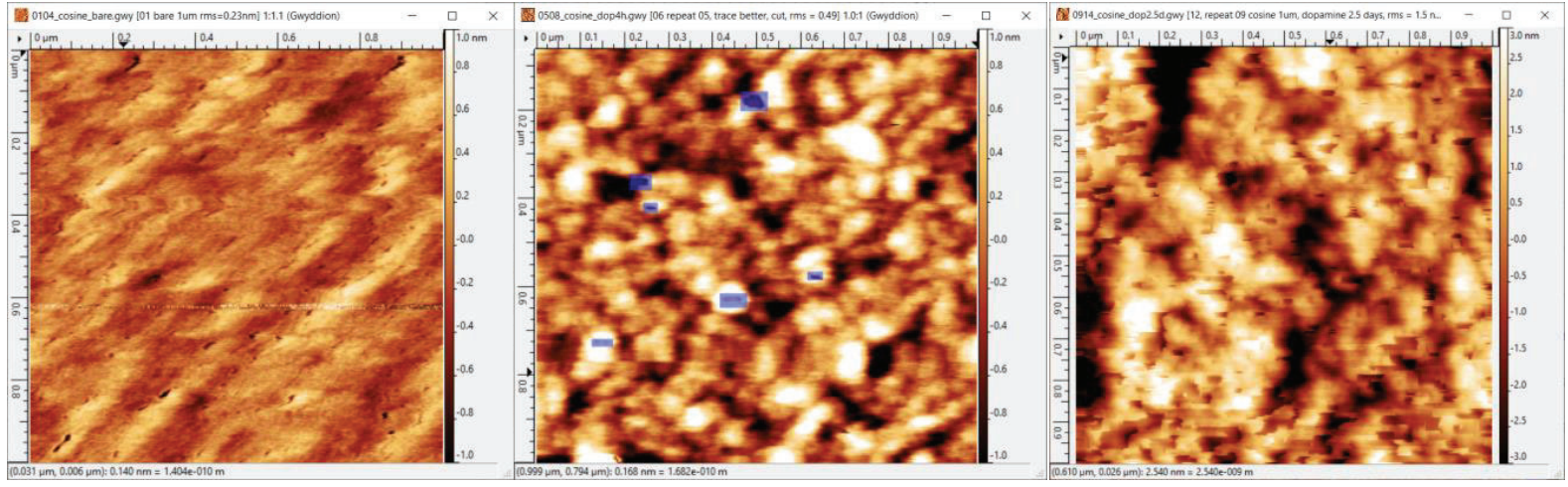

Figure 6: AFM $1 \mu \mathrm{m}$ side. Left: $\mathrm{Ir}+\mathrm{Cr}$ coated $\mathrm{Si}$ substrate $(\mathrm{rms}=2.3 \AA)$; Center: dopamine deposition 4 hours $(\mathrm{rms}=4.9 \AA)$; Right: dopamine deposition 2.5 days $(\mathrm{rms}=15 \AA)$; Data analysis by Gwyddion software ${ }^{33}$.

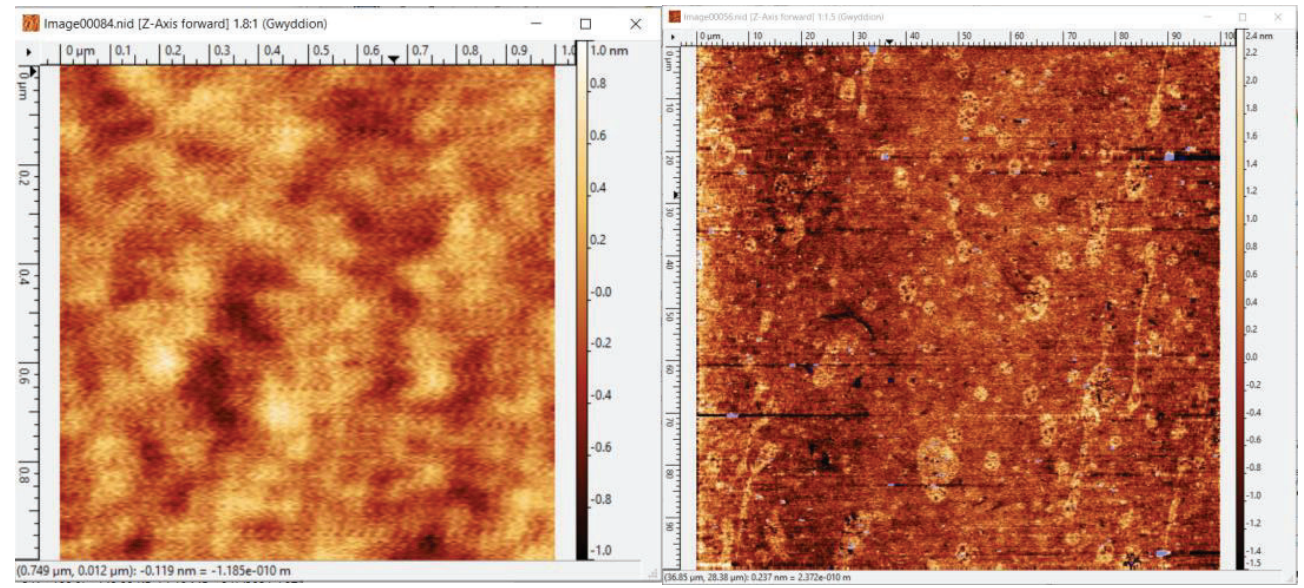

Figure 7: AFM scan for for 24 hours deposition dopamine. Left: $1 \mu \mathrm{m}$ size, rms $2.3 \AA$. Right: $100 \mu \mathrm{m}$ size, rms $6.0 \AA$. 


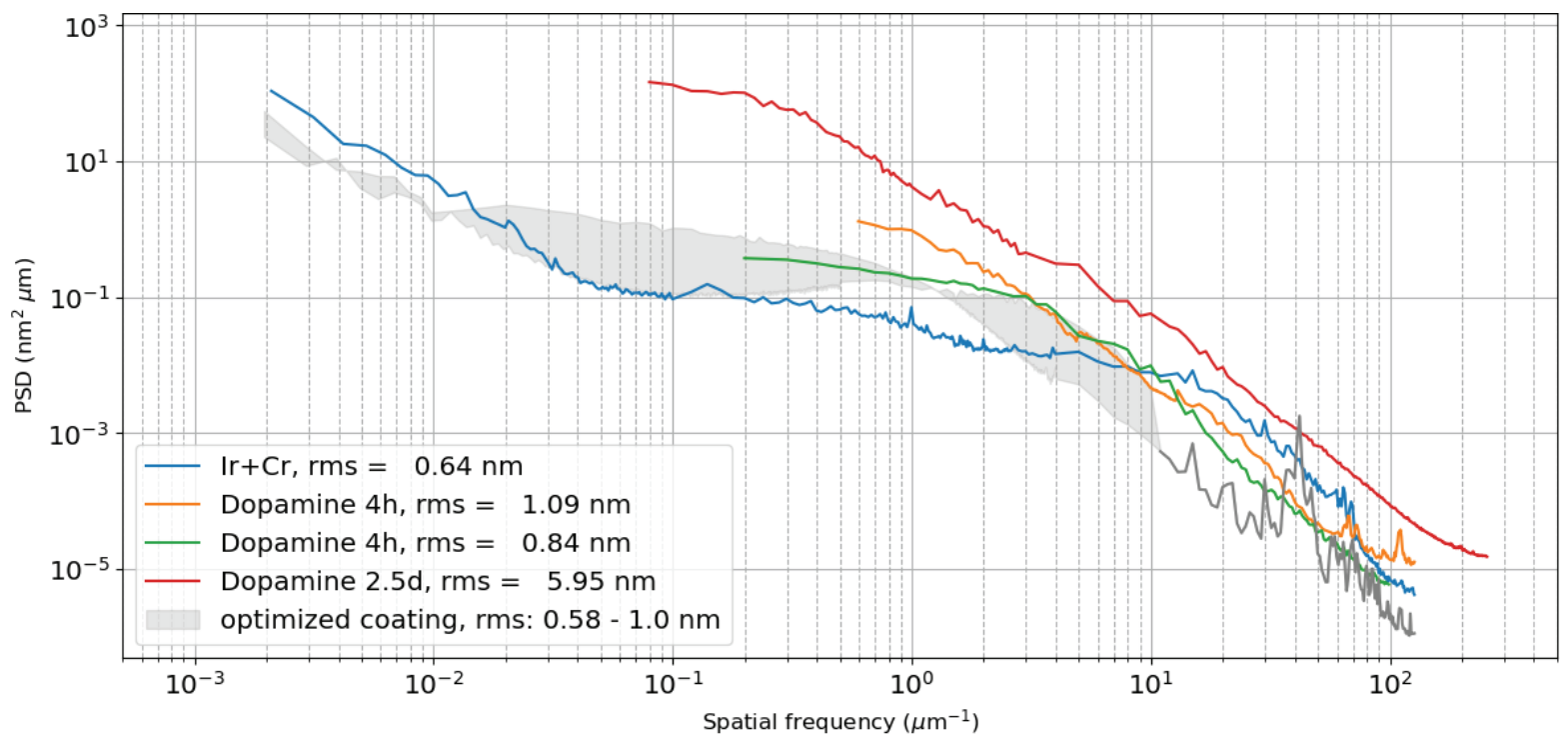

Figure 8: Comparison of PSDs for dopamine overcoatings in tests (4h, 2.5d) and final deposition (optimized coating). The gray area shows the range for PSD as estimated from different measurement points and samples. Our best estimate for the full PSD is shown in figure 9.

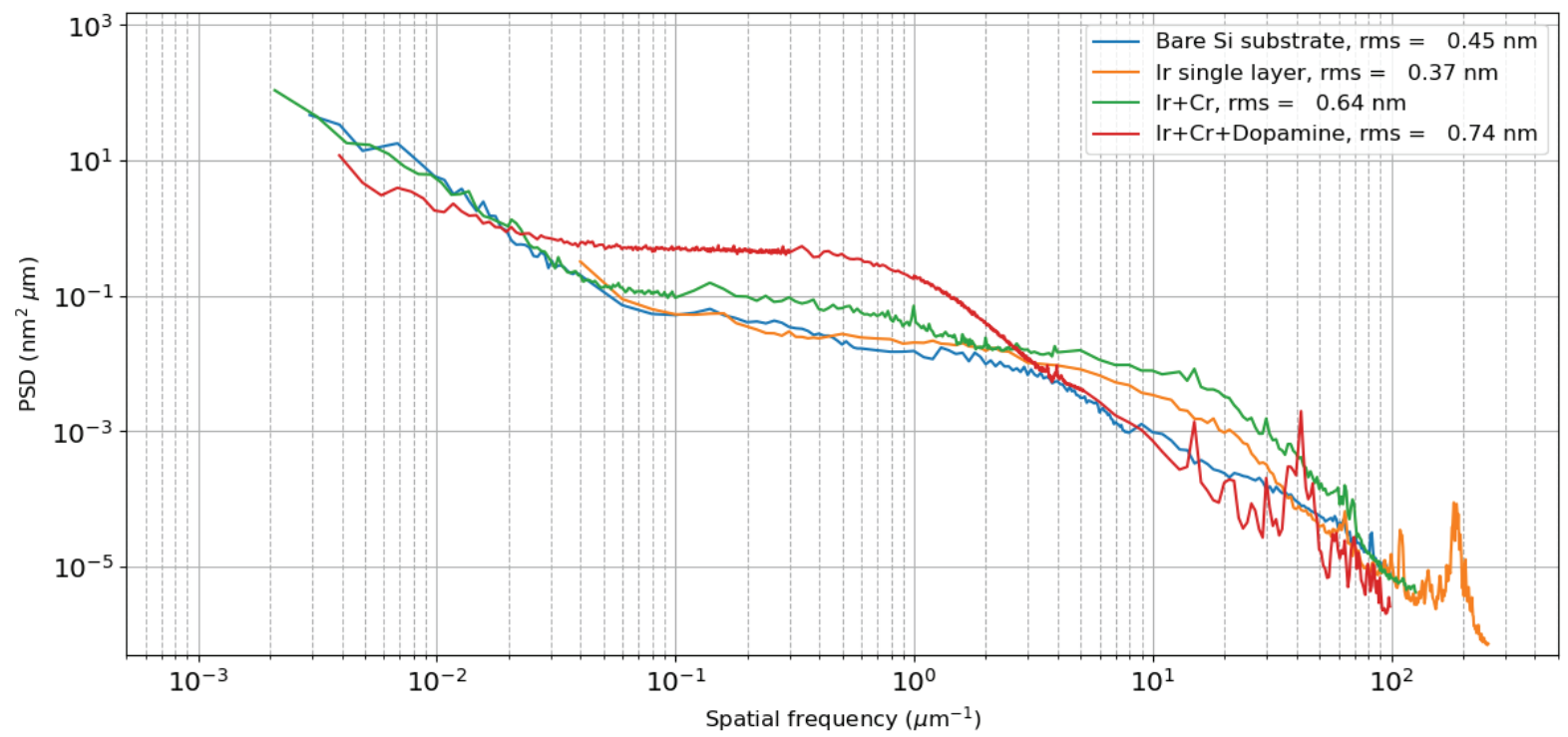

Figure 9: Comparison of PSDs obtained from surface metrology data for the sequence of coatings from substrate to overcoating. Red line represents a realistic estimate for the optimized dopamine coating, as obtained by different samples and points of measurement. rms values in legend are obtained from the full frequency ranges.

\section{OVERCOATINGS ON GOLD}

We have deposited Carbon-like coatings on gold substrate by liquid-phase deposition of self-assembled layers of thiol and silane molecules. Gold is a material of interest, as it is the common choice for the realization of monolithic shells by means of nickel replica process, for which acts as release agent between the mirror shell and the mandrel on which the shell is electroformed (in particular, this method will be used for the realization of the optics for the eXTP mission by CAS). The bilayer approach was adopted in order to achieve the desirable coating thickness of 6-10 nm. In fact, SAM coatings are usually extremely thin layers $(<2-3 \mathrm{~nm})$ but strongly depending on precursor molecules, deposition technique and parameters $^{34-36}$. Here, long alkyl chains thiol and chlorosilanes were employed for the bilayer coating. In particular, a double-functional thiol was employed for the first layer, owing to its strong binding affinity to gold substrate by the thiol group and crosslinking capability through the triethoxysilane ending group ${ }^{37,38}$. Substrates were cleaned by rinsing in 
acetone and 2-propanol and then immersed in a $2 \mathrm{mM}$ 11-Mercaptoundecyltrimethoxysilane $\left(\mathrm{C}_{14} \mathrm{H}_{32} \mathrm{O}_{3} \mathrm{SSi}\right.$, here named M11, provided by company Sigma Aldrich) in ethanol for $24 \mathrm{~h}$ for the deposition of the first layer. Second layer was deposited by immersing the M1 1-coated substrate in a $2 \mathrm{mM}$ solution of Trichloro(octadecyl)silane $\left(\mathrm{CH}_{3}\left(\mathrm{CH}_{2}\right)_{17} \mathrm{SiCl}_{3}\right.$, here named OTS, provided by Gelest company) in toluene for other $24 \mathrm{~h}$.

Nominal values for density from provider's data sheets are $0.98 \mathrm{~g} / \mathrm{cm}^{3}$ for OTS and $0.96 \mathrm{~g} / \mathrm{cm}^{3}$ for M11, the expected thickness of the coating was estimated from preliminary test samples, to be respectively $2.5 \mathrm{~nm}$ and $4.5 \mathrm{~nm}$ for M11 and OTS, against a theoretical value of about $2 \mathrm{~nm}$ for both monolayers, consistently with our expectations of having some amount of uncontrolled growth, especially for OTS on top on the more ordered M11.

\section{Thickness characterization}

Ellipsometric data were collected in different positions of flat samples. Thickness was set as the only free parameter, and the film was modelled according to a two parameter Cauchy model. $\mathrm{A}=1.414, \mathrm{~B}=0.01, \mathrm{C}=0$. Four points at increasing distance from the center in different radial directions, were measured. Resulting values, in order of distance from center to edge are 9.0, 9.7, 8.6, $7.4 \mathrm{~nm}$, indicating a good uniformity and small errors in fit of data. Measurements on shell fragments gave similar values (in the range 6-8 $\mathrm{nm}$ ), but it was necessary to include the angular dispersion due to the non planar shape of samples. The measured value is slightly higher than the expected one, which can be due to uncertainty in the measurement and simplified modelling, or to a partially unordered growth of the second layer, more sensitive to deposition conditions. We don't have yet data for surface topography of this samples, as sample production and characterization was done before the acquisition of an AFM in our facilities. Work is ongoing for the production and complete characterization of new samples.
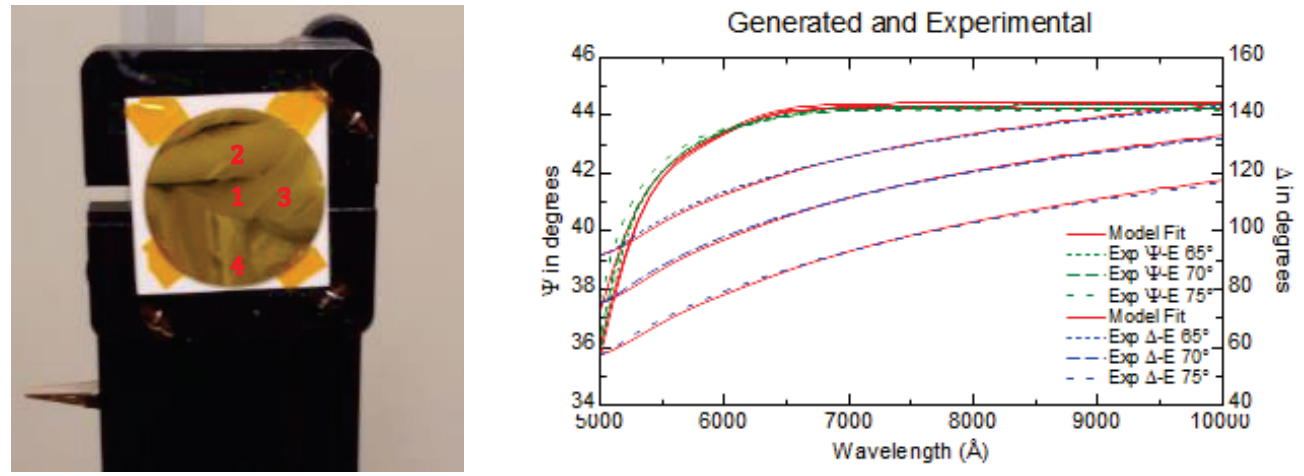

Figure 10: Left pane: setup for ellipsometric measurement showing the measurement points. Right pane: Comparison of ellipsometric data related to the thinnest (\#4) and thickest (\#2) point. Model fit worsen towards blue but the fitting error is in any case smaller than the difference between ellipsometric data for the two spots.

\section{CONCLUSIONS AND EXPECTED DEVELOPMENT}

We have deposited iridium-chromium bilayers coatings on substrates representative of X-ray mirrors, like the silicon plates that will be used for the ATHENA pore optics, measuring surface topography for each layer and extracting PSD information over a broad range of spatial frequencies. While roughness slightly increases (as expected) along the layers stack, it is shown that all layers maintains an acceptable roughness, with chromium layer having a rms below $4 \AA^{\circ}$, over $10^{-}$ ${ }^{2}$ to $10^{2} \mu \mathrm{m}^{-1}$ frequency range, and $6.4 \AA$ over a broader frequency range (down to $210^{-3} \mu \mathrm{m}^{-1}$ ) with a rather flat PSD in the central part of the spectral range under investigation. The roughness values are in good agreement with the rms roughness of 3-5 A estimated from X-ray reflectivity fit. Surface quality of the metallic coatings are very similar between the two facilities used for the coating deposition for all thickness values of 25, 60 and $100 \AA$.

These substrates were used for the deposition of a dopamine overcoating, which was optimized to give a roughness just slightly above the one of the substrates (between 6 and $10 \AA$ over the extended frequency range with the value depending on the sample and measurement point). The coating shows uniform structure on the largest scales (AFM over $100 \mu \mathrm{m}$ scale and 10x optical profilometer). The thickness was roughly evaluated by scratch test, giving results in the valid range (5-10 $\mathrm{nm})$.

The collected PSD data will be used to model the scattering properties and infer the X-ray properties. We plan to characterize the coated samples in X-rays after a more thorough characterization of film properties. 
We also produced first samples of bilayer carbon-based coatings of thiols and silans, on gold, representative of optics realized by nickel-replica process, like the one that will be used for eXTP mirrors, and characterized their thickness using spectroscopic ellipsometry. The results are in line with the expectations even if slightly above the design values (a thickness between 7.5 and $9.0 \mathrm{~nm}$ was obtained vs an expected thickness of $6.5 \mathrm{~nm}$ ), that might indicate the needs for a more accurate optimization of the deposition process.

\section{REFERENCES}

[1] B. Aschenbach, X-ray Telescopes, Reports on Progress in Physics, Volume 48, pp. 579-629 (1985).

[2] P. Gorenstein, "Grazing incidence telescopes for x-ray astronomy," Opt. Eng. 51(1) 011010 (2012)

[3] M. Bavdaz, E. Wille, M. Ayre, I. Ferreira, B. Shortt, et al., "Development of the ATHENA mirror", Proc. SPIE 10699, Space Telescopes and Instrumentation 2018: Ultraviolet to Gamma Ray, 106990X (2018)

[4] D. Della Monica Ferreira, S. Massahi, F. E. Christensen, B. Shortt, M. Bavdaz, et al., "Design, development, and performance of $x$-ray mirror coatings for the ATHENA mission”, Proc. SPIE 10399, Optics for EUV, X-Ray, and GammaRay Astronomy VIII, 1039918 (2017)

[5] J. A. Gaskin, A. Dominguez, K. Gelmis, J. Mulqueen, D. Swartz, et al., "The Lynx X-ray Observatory: concept study overview and status", Proc. SPIE 10699, Space Telescopes and Instrumentation 2018: Ultraviolet to Gamma Ray, $106990 \mathrm{~N}(2018)$

[6] Giovanni Pareschi, Vincenzo Cotroneo, Daniele Spiga, Dervis Vernani, Marco Barbera, Maria Antonella Artale, Alfonso Collura, Salvatore Varisco, Gabriele Grisoni, Giuseppe Valsecchi, Barbara Negri, "Astronomical soft x-ray mirrors reflectivity enhancement by multilayer coatings with carbon overcoating," Proc. SPIE 5488, UV and Gamma-Ray Space Telescope Systems, (2004)

[7] V. Cotroneo, D. Spiga, M. Barbera, R. Bruni, K. Chen, C. Marcelli, G. Pareschi, S. Romaine, Y. D. Zhao, L. Zheng, Z. Y. Wu, "Carbon overcoatings for soft $x$-ray reflectivity enhancement," Proc. SPIE 6688, Optics for EUV, X-Ray, and Gamma-Ray Astronomy III, 66880U (2007)

[8] V. Cotroneo, D. Spiga, R. Bruni, W. Burkert, M. Freyberg, G. Hartner, G. Pareschi, S. Romaine, "New developments in light material overcoating for soft $x$-ray reflectivity enhancement," Proc. SPIE 7011, Space Telescopes and Instrumentation 2008: Ultraviolet to Gamma Ray, 701119 (2008)

[9] Desiree Della Monica Ferreira, Sonny Massahi, Finn E. Christensen, Brian Shortt, Marcos Bavdaz, Maximilien J. Collon, Boris Landgraf, Nis C. Gellert, Jakob Korman, Paschalis Dalampiras, Ida F. Rasmussen, Ifikratis Kamenidis, Michael Krumrey, Swenja Schreiber, "Design, development, and performance of x-ray mirror coatings for the ATHENA mission," Proc. SPIE 10399, Optics for EUV, X-Ray, and Gamma-Ray Astronomy VIII, 1039918 (2017)

[10] Desirée Della Monica Ferreira, Sara Svendsen, Sonny Massahi, Atefeh Jafari, Lan M. Vu, Jakob Korman, Nis C. Gellert, Finn E. Christensen, Shima Kadkhodazadeh, Takeshi Kasama, Brian Shortt, Marcos Bavdaz, Maximilien J. Collon, Boris Landgraf, Michael Krumrey, Levent Cibik, Swenja Schreiber, Anja Schubert, "Performance and stability of mirror coatings for the ATHENA mission," Proc. SPIE 10699, 106993K (2018)

[11] Y. Yang, O. Huang, H. Ni, et al., "High reflectance broadband thin films used in x-ray telescopes", Proc. SPIE, Volume 10691, id. 106910S. (2018)

[12] S. Zhang, et al., "The enhanced X-ray Timing and Polarimetry mission-eXTP", SCIENCE CHINA Physics, Mechanics \& Astronomy, 029502(2019), Vol 62 (2019)

[13] Veronika Stehlíkova, Thorsten Döhring, Tobias Schäfer, Manfred Stollenwerk, Peter Friedrich, Vadim Burwitz, Gisela Hartner, Miranda Bradshaw, Yingyu Liao, and Carlo Pelliciari: "X-ray reflectivity measurements at chromiumiridium tri-layer coatings", Proc. SPIE 11119, 111191L (2019)

[14] V. Stehlikova, A.-C. Probst, O. Nentvich, M. Urban, T. Döhring, L. Sieger and R. Hudec, "Study of multiple layers coatings for X-ray mirrors", Contrib. Astron. Obs. Skalnaté Pleso 48, $488-497$ (2018)

[15] Stefano Casalini, Carlo Augusto Bortolotti, Francesca Leonardi and Fabio Biscarini, "Self-assembled monolayers in organic electronics", Chem. Soc. Rev., 2017, 46, 40 (2017)

[16] Palladino, P., Bettazzi, F., and Scarano, S., "Polydopamine: surface coating, molecular imprinting, and electrochemistry - successful applications and future perspectives in (bio)analysis", Anal Bioanal Chem 411, 4327-4338 (2019)

[17] Yanlan Liu, Kelong Ai and Lehui Lu, "Polydopamine and Its Derivative Materials: Synthesis and Promising Applications in Energy, Environmental, and Biomedical Fields", Chem. Rev. 2014, 114, 5057-5115

[18] Vladsinger - CC BY-SA 4.0, https://commons.wikimedia.org/w/index.php?curid=68470397

[19] G. Pareschi, M. Civitani, G. Sironi, Y. Yang, V. Cotroneo, G. Valsecchi, L. Magagnin, T. Döhring, M. Bradshaw, V. Burwitz, C. Pelliciari, "Soft x-ray reflectivity-enhancement in astronomical telescopes via overcoatings: alternative materials and deposition methods", Proc. SPIE 11119, Optics for EUV, X-Ray, and Gamma-Ray Astronomy IX, 111190S (2019) 
[20] David L. Windt, "IMD—Software for modeling the optical properties of multilayer films", Computers in Physics 12:4, 360-370 (1998)

[21] S. Romaine, R. Bruni, P. Gorenstein, and Z. Zhong, "Measurements of the hard-x-ray reflectivity of iridium", Appl. Opt. 46, 185-189 (2007)

[22] A. Ames, D. Ampleford, C. Bourdon, R. Bruni, K. Kilaru, B. Kozioziemski, M. Pivovaroff, B. Ramsey, S. Romaine, J. Vogel, C. Walton, M. Wu, "Characterization of multilayer coated replicated Wolter optics for imaging $x$-ray emission from pulsed power," Proc. SPIE 10399, Optics for EUV, X-Ray, and Gamma-Ray Astronomy VIII, 103991X (2017)

[23] Adrian Ivan, Ricardo J. Bruni, Kyung Wha Byun, Finn Erland Christensen, Paul Gorenstein, Suzanne E. Romaine, "Design and optimization of multilayer coatings for hard x-ray mirrors," Proc. SPIE 3773, X-Ray Optics Design, Performance, and Applications, (1999)

[24] A. Ames, R. Bruni, V. Cotroneo, R. Johnson-Wilke, T. Kester, P. Reid, S. Romaine, S. Tolier-McKinstry, R. H. T. Wilke, "Using iridium films to compensate for piezo-electric materials processing stresses in adjustable $x$-ray optics," Proc. SPIE 9603, Optics for EUV, X-Ray, and Gamma-Ray Astronomy VII, 96031I 2015)

[25] Anne-Catherine Probst, Thomas Begou, Thorsten Döhring, Sebastian Zeising, Manfred Stollenwerk, Johannes Stadtmüller, Florian Emmerich, and Julien Lumeau: "Coating stress analysis and compensation for iridium based X-ray mirrors”, Applied Optics 57(29), 8775-8779 (2018)

[26] Thorsten Döhring, Anne-Catherine Probst, Manfred Stollenwerk, Florian Emmerich, Veronika Stehlíková, Adolf Inneman: "Prototyping iridium coated mirrors for X-ray astronomy", Proceedings of SPIE 10235, 10235-04 (2017)

[27] A. Büttner, A.-C. Probst, F. Emmerich, C. Damm, B. Rellinghaus, T. Döhring, M. Stollenwerk: "Influence of Sputtering Pressure on the Microstructure and Layer Properties of Iridium Thin Films", Thin Solid Films 662, 41-46 (2018)

[28] Anne-Catherine Probst, Manfred Stollenwerk; Florian Emmerich; Andre Büttner; Sebastian Zeising; Johannes Stadtmüller; Franziska Riethmüller; Veronika Stehlíková; Mingwu Wen; Laura Proserpio; Christine Damm; Bernd Rellinghaus; Thorsten Döhring: "Influence of sputtering pressure on the nanostructure and the X-ray reflectivity of iridium coatings", Surface \& Coatings Technology 343, 101-107 (2018)

[29] Anne-Catherine Probst, Thorsten Döhring, Manfred Stollenwerk, Laura Proserpio, Mingwu Wen: "Iridium coatings for space based X-ray optics", Proceedings of the International Conference on Space Optics, Biarritz, France, 18 - 21 October 2016, Paper FP_IC_177 (ICSO 2016)

[30] Jürgen Liebscher "Chemistry of Polydopamine - Scope, Variation, and Limitation" European Journal of Organic Chemistry Volume 2019, Issue 31-32 p. 4976-4994 (2019)

[31]Ji Hyun Ryu, Phillip B. Messersmith, and Haeshin Lee "Polydopamine Surface Chemistry: A Decade of Discovery" ACS Applied Materials \& Interfaces 201810 (9), 7523-7540, DOI: 10.1021/acsami.7b19865

[32] Qiang Wei, Fulong Zhang, Jie Li, Beijia Lia and Changsheng Zhao, "Oxidant-induced dopaminepolymerization for multifunctional coatings", Polym. Chem., 2010,1, 1430-1433 (2010)

[33] David Nečas, Petr Klapetek, "Gwyddion: an open-source software for SPM data analysis", Cent. Eur. J. Phys. 10(1) 181-188 (2012)

[34] Touraj Manifar, Asad Rezaee, Mehdi Sheikhzadeh, Silvia Mittler, "Formation of uniform self-assembly monolayers by choosing the right solvent: OTS on silicon wafer, a case study", Applied Surface Science, Volume 254, Issue 15, 2008, Pages 4611-4619, ISSN 0169-4332 (2008)

[35] Sunhyung Lee, Takahiro Ishizaki, Nagahiro Saito and Osamu Takai "Effect of Reaction Temperature on Growth of Organosilane Self-Assembled Monolayers", Japanese Journal of Applied Physics, Volume 47, Number 8R, 6442 (2008)

[36] Noémi Rozlosnik, Michael C. Gerstenberg, and Niels B. Larsen "Effect of Solvents and Concentration on the Formation of a Self-Assembled Monolayer of Octadecylsiloxane on Silicon (001)", Langmuir 2003, 19, 4, 1182-1188 (2003)

[37] Xue, Y., Li, X., Li, H. et al. "Quantifying thiol-gold interactions towards the efficient strength control" Nat Commun 5, 4348 (2014)

[38] C. Vericat, M. E. Vela, G. Benitez, P. Carrob and R. C. Salvarezza, "Self-assembled monolayers of thiols and dithiols on gold: new challenges for a well-known system” Chem. Soc. Rev., 2010,39, 1805-1834 (2010) 